\title{
Moisture Management Properties of Textiles and Its Evaluation
}

\author{
Mayur Basuk ${ }^{1}$, Mrinal Choudhari ${ }^{1}$, Saptarshi Maiti ${ }^{2}$, RV Adivarekar ${ }^{2 *}$ \\ ${ }^{1}$ Wool Research Association, Thane
}

${ }^{2}$ Department of Fibres and Textile Processing Technology, Institute of Chemical Technology, India

Submission: January 25, 2018; Published: February 28, 2018

*Corresponding author: RV Adivarekar, Department of Fibres and Textile Processing Technology, Institute of Chemical Technology, Matunga, Mumbai, India Tel: 022-33612801/09833408172; Email: rv.adivarekar@ictmumbai.edu.in

\begin{abstract}
Moisture management is one of the key performance criteria in today's apparel industry, which decides the comfort level of any fabric. The consumer's demand for comfort performance of the garment is on high rise and inevitably apparel manufacturers have been compelled to shift their attention towards the high-performance of moisture management technology and market. This review encompasses the necessity of moisture management in textile apparel, aims of development of moisture management fabrics, technical approach towards moisture management, desired attributes of moisture management fabrics, the route to moisture management, various concepts of moisture managing textiles, developments in moisture management techniques and functional fields of application of moisture management technique.
\end{abstract}

Keywords: Moisture management; Apparel; Comfort; Development; Application

Abbreviations: MMT: Moisture Management Tester; PCM: Phase Change Materials; MWR: Maximum Wetted Radius; OMMC: Overall Moisture Management Capacity

\section{Introduction}

Technical textiles are the textile materials and products manufactured primarily for their technical and performance properties rather than their aesthetic or decorative characteristics. One of the most important branch of technical textiles is Sportech, used in sports and leisure. The comfort properties of sportswear are largely determined by its moisture management properties. In India, Sportech market is on high growth with increasing popularity [1,2].

There is a rapid change in fashion and apparels worldwide. Whether it is woven or knitted, the demand is for something new and different in the world of high fashion. As the worldwide business gears to tackle the challenges, product diversification has become the key to global opportunities. The main factors affecting consumer's selection of garments are aesthetic, appearance and fashion. Besides these factors, comfort properties of garments during usage cannot be neglected. Now-a-days, consumers have become much more demanding in terms of properties of clothing, particularly those of leisure and sportswear. One of the textile properties that have steadily gained importance among increasingly well-informed consumers is breathability. To maintain the state of comfortness, clothing must be designed to allow body's heat balance to be maintained over a wide range of environmental conditions. It should fulfil this function without inhibiting the evaporation of humidity caused by perspiration, and thus not interfering with the temperature regulation of the body [3]. Moisture management can be defined as the controlled movement of water vapor and liquid water (perspiration) from the surface of skin to atmosphere through the textile substrate. This action prevents perspiration remaining next to skin. Wearing garments that transport moisture and evaporate it quickly actually enhance body's ability to cool itself [4].

The main aim to develop moisture management fabrics is; to transport the humidity to the atmosphere as fast as possible, to evaporate it and making the skin feel dry. Moisture management includes the use of microfiber technology or the application of various softening finishes like silicones at the molecular level to enhance both hydrophobic and hydrophilic properties of a fabric [5]. Water-resistant and moisture-permeable materials may be divided into three main categories: high-density fabrics, resincoated materials and film-laminated materials.

Some standards and test methods can be employed to evaluate the fabric's simple absorbency and wicking properties. The liquid strike-through time of non-wovens can be tested according to ISO 9073-8. The Moisture Management Tester (MMT) is an instrument to measure the dynamic liquid transport properties of textiles such as knitted and woven fabrics in three dimensions: 
o Absorption Rate - Moisture absorbing time of the fabric's inner and outer surfaces.

o One-way Transport Capability - Liquid moisture oneway transfer from the fabric's inner surface to outer surface.

o Spreading/Drying Rate - Speed of liquid moisture spreading on the fabric's inner and outer surfaces.

\section{Discussion}

\section{Principle of measurement}

The liquid moisture management properties of textiles are evaluated by placing a fabric specimen between two horizontal (upper and lower) electrical sensors each with seven concentric pins in a MMT. A predetermined amount of test solution that aids the measurement of electrical conductivity changes are dropped onto the center of the upward-facing test specimen surface. The test solution is free to move in three directions. During this test, changes in electrical resistance of specimen are measured and recorded. The electrical resistance is used to calculate fabric liquid moisture content changes that quantify dynamic liquid moisture transport behavior of the specimen. The summary of the measured results are used to grade the liquid moisture management properties of any fabric by using predetermined indices.

MMT is designed to sense, measure and record the liquid moisture transport behavior in multiple directions and can distinguish seven major types of fabrics. A series of indexes are defined and calculated to characterize liquid moisture management performance of the test specimen. The measurement is based on water resistance, water repellency and water absorption characteristics of the fabric structure, including the fabric's geometric and internal structure along with the wicking characteristics.

\section{Requirement for moisture management}

Comfort can be defined as a pleasant physical, physiological and psychological equilibrium state between the human beings and environment. For a person engaged in normal routine indoor activity, energy expended is generally around 50watts/square meter/hour. The metabolic heat generated is readily dissipated through the clothing as sweat. At rest, a human body usually gives off, about $60 \mathrm{ml} / \mathrm{hr}$ of water vapor at ambient conditions. Moderate exertion (walking) increases that amount to about $450 \mathrm{ml} / \mathrm{hr}$. During sporting activity, e.g. tennis or cycling, the metabolic heat increases to about six times and perspiration nearly 14 times $(840 \mathrm{ml} / \mathrm{hr}$ ). During sweating, human body humidity is more or less absorbed by the textile apparels. If humidity remains in the fabric and is not transported to the surface for evaporation, cooling cannot occur. The body warms up and even more sweat is produced. During hard physical activity body sweats and in conventional clothing like cotton, the moisture gets trapped. The sports and leisure wears exert a barrier for efficient transfer of excess heat resulting in a rise in core body temperature and skin temperature greater than 37 ${ }^{\circ} \mathrm{C}$ that increases sweating. The excess heat moistens the fabric, reducing the body heat and making the wearer tired. So the fabric worn next to skin should have two important properties. The initial and the foremost property is to evaporate perspiration from the skin surface and the second property is to transfer moisture in the atmosphere. Thus, moisture management fabric is very useful if worn at the time of exercising to keep the skin dry making the wearer feel comfortable.

\section{Approaches for development of Moisture Management}

$100 \%$ cellulosic fiber garments are widely used for general sports clothing and street wear, but the only fabrics actively promoted for high performance sportswear are made from synthetic fibers. Consumers and sportswear manufacturers have a view that cellulosic fibers are unsuitable in sportswear industry for high activity where sweat production needs to be dealt with. The reasons for this view of cellulosic fabrics are real and need to be addressed if the use of cellulosic fibers in sportswear is to be increased. Cellulosic fabrics absorb water into the fiber structure and become heavy. This leads to stretching of the fabric, sticking to the skin and when activity ceases, the fabric may feel cold against the skin. Higher levels of moisture absorbed in the fabric means longer drying times. However, cellulosic fabrics are generally perceived to be more comfortable than synthetic fabrics when worn for normal dayto-day activities. They are preferred for a wide range of apparel fabrics where visual aesthetics, handle and comfort are essential.

In order to deliver these positive properties of cellulosic fibers and to eliminate the negative aspects of performance, a new approach is required. A successful cellulosic fabric will need to have much lower absorption capacity than a $100 \%$ cellulosic but must also deliver better visual aesthetics along with excellent moisture handling capability. Hydrophilic fabrics and waterproof breathable fabrics are some advances in the field of moisture management in textiles [6-9].

\section{History of moisture management}

Human body acts like perfect air conditioning. This sophisticated system reliably regulates our body temperature to a constant $37{ }^{\circ} \mathrm{C}$, which is the optimum temperature for maximum performance and comfort. An essential control mechanism of this system is the release of moisture at the right moment to protect the body from overheating. To maintain the body's optimum performance, a textile worn next to skin has to support this regulative mechanism. A textile should work as our own skin. An important feature of any fabric is how it transports this water out of the body surface so as to make the wearer feel comfortable. From the comfort point of view, moisture transmission through textile material both in liquid and vapour forms are equally important. Liquid moisture flow through textile materials is controlled by two processes-wetting and wicking. 
Wetting is the initial process, involved in fluid spreading; it is controlled by the surface energies of the involved solid and liquid. In case of textile material as soon as water wets the fiber, the water enters the inter fiber capillary channel and is dragged along by the action of capillary pressure. Wetting, wicking and moisture vapor transmission properties are critical aspects for assessing the comfort performance of textile products. Wetting is the initial process involved in fluid spreading. The fiber-air interface is replaced with a fiber-liquid in this process. In the case of clothing with high wicking properties, moisture coming from the skin is spread throughout the fabric offering a dry feeling and spreading of the liquid enables moisture to evaporate easily.

\section{Developments in moisture management techniques}

The term "moisture management" is often used as an advertising slogan. However, ideas differ among textile manufacturers as to how to achieve an optimized moisture management. In order to bring about the different effects, a suitable fiber material is used or a subsequent finishing is applied. It is also possible to combine specialized fibers and finishings.

Hydrophobic textiles: Absorb only a little amount of humidity which can lead to insufficient transmis $\neg$ sion of humidity away from the skin and to an unpleasant feeling of dampness. Furthermore, the water which is not transported to the outer surface is no longer available for cooling of the body.

Hydrophilic textiles: Hydrophilic Textiles are known for their greater capacity to absorb humidity. Emerging liquid is absorbed efficiently and transported to the skin surface for evaporation. However, after exercise, a large amount of liquid has to evaporate to cause stronger cooling and freezing.

Hydrophobic-Hydrophilic textiles: Hydrophobic-Hydrophilic Textiles are designed to transport humidity rapidly from the skin and evaporate it outside. The special construction of the material enables trans $\neg$ portation of humidity from inside to outside of the substrate. The two-sidedness of the fabric is either attained by processing different materials during manufacturing or by varied coatings of the fabric surfaces.

Micro fibers: Micro Fibers by virtue of their extreme fineness, form especially small gaps and have a big surface area. This leads to high capillary effect for the transportation of humidity, and rapid evaporation.

Special fibers: Special Fibers are designed to increase the capillary force and the humidity transportation, by means of special profiles. The larger surface area of these fibers also serves to promote evaporation.

Waterproof-Breathable textiles: Waterproof-Breathable Textiles provide protection from the environmental factors like wind, rain and loss of body heat. Waterproof fabric completely prevents the penetration and absorption of liquid water. The term breathablility implies that the fabric is actively ventilated.
Breathable fabrics passively allow water vapor to diffuse through them yet prevent the penetration of liquid water. High functional fabrics support active sportswear with importance placed on high functions as well as comfort.

Spacer fabrics: Spacer Fabrics consists of two separate fabric webs, which are joined together by spacer threads or fibers of varying rigidity. The intermediate zone creates a layer of air, having an insulating and thermoregulatory effect. An important advantage is its low weight in proportion to large volume. The application areas of spacer fabric are unlimited ranging from healthcare, safety, military, automotive, aviation and fashion. Currently it is being largely used for functional clothing comprising sports shoes, bra cups, shoulder pads, knee and elbow protectors etc. [10].

Phase change materials (PCM): Phase Change Materials are materials that have the distinctive capacity to soak and emit heat energy without altering the temperature. They possess different freezing and melting points and when mixed in a microcapsule, accumulate and release heat energy and maintaining the temperature range of $30-34^{\circ} \mathrm{C}$, which is very comfortable for the body. PCM microcapsules can create small, transitory heating and cooling effects in garment layers when the temperature of the layers reaches the PCM transition temperature [11].

Table 1: Different types of Fabric \& its Characteristics.

\begin{tabular}{|c|c|c|}
\hline Sr. No. & Type of Fabric & Properties \\
\hline 1. & Water Proof Fabric & $\begin{array}{c}\text { Very slow absorption } \\
\text { Slow spreading } \\
\text { No one-way transport, no } \\
\text { penetration }\end{array}$ \\
\hline 2. & $\begin{array}{l}\text { Water Repellent } \\
\text { Fabric }\end{array}$ & $\begin{array}{c}\text { No wetting } \\
\text { No absorption } \\
\text { No spreading } \\
\text { Poor one-way transport without } \\
\text { external forces }\end{array}$ \\
\hline 3. & $\begin{array}{l}\text { Slow Absorbing and } \\
\text { Slow Drying Fabric }\end{array}$ & $\begin{array}{c}\text { Slow absorption } \\
\text { Slow spreading } \\
\text { Poor one-way transport }\end{array}$ \\
\hline 4. & $\begin{array}{l}\text { Fast Absorbing and } \\
\text { Slow Drying Fabric }\end{array}$ & $\begin{array}{l}\text { Medium to fast wetting } \\
\text { Medium to fast absorption } \\
\text { Small spreading area } \\
\text { Slow spreading } \\
\text { Poor one-way transport }\end{array}$ \\
\hline 5. & $\begin{array}{l}\text { Fast Absorbing and } \\
\text { Quick Drying Fabric }\end{array}$ & $\begin{array}{l}\text { Medium to fast wetting } \\
\text { Medium to fast absorption } \\
\text { Large spreading area } \\
\text { Fast spreading } \\
\text { Poor one-way transport }\end{array}$ \\
\hline 6. & $\begin{array}{l}\text { Water Penetration } \\
\text { Fabric }\end{array}$ & $\begin{array}{c}\text { Small spreading area } \\
\text { Excellent one-way transport }\end{array}$ \\
\hline 7. & $\begin{array}{c}\text { Moisture } \\
\text { Management Fabric }\end{array}$ & $\begin{array}{l}\text { Medium to fast wetting } \\
\text { Medium to fast absorption } \\
\text { Large spread area at bottom surface } \\
\text { Fast spreading at bottom surface } \\
\text { Good to Excellent one-way transport }\end{array}$ \\
\hline
\end{tabular}


Pine cone effect: Pine cones on the trees are seemed to be closed but when they fallout from the tree it gets opened to release the seeds. The scales of the pine cone get opened as they are made up of two layers of stiff fibers running in different directions. As the cone dries out, the scales inside get expanded more than outside, causing the outer scales to bend outwards, releasing the seeds inside. The pine cone effect is a technology designed to offer a solution to the discomfort sensations caused by the moisture which is built up due to the changing temperature in clothing. It is impossible to predict the Indian temperature, humidity and activity level so that to accommodate in a selection of clothing to ensure comfort. There may be the other ways of producing ventilating or insulating fabrics by addition or removal of layers of clothing, addition of garment parts or by introduction of ventilating features to the garments but the limited availability of space and the wearer's ability makes discomfort sensations (Table 1). To overcome this, the pine cone effect is employed in clothing which is advantageous over others [12].

\section{Measurement and evaluation of moisture management properties of textiles}

Below mentioned apparatus and accessories are required to measure moisture management properties of textiles.

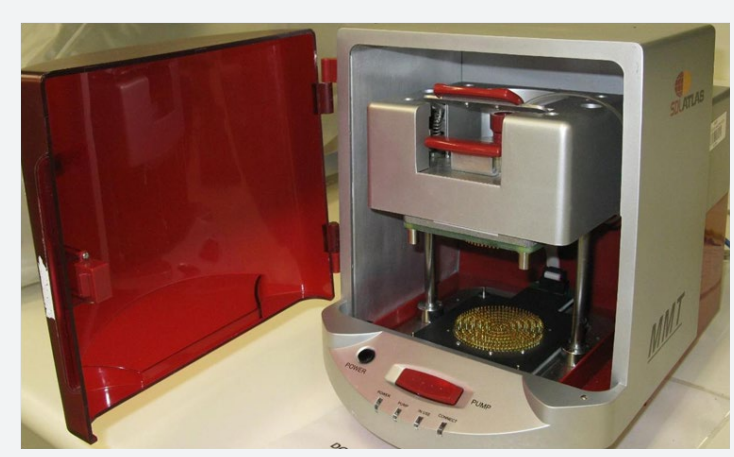

Figure 1: Moisture Management Tester (MMT).

o Moisture Management Tester

o $\quad$ Computer with MMT software installed

o Distilled water

o $\quad$ Sodium chloride solution $(0.9 \% \mathrm{NaCl})$ o Conductivity meter to check the conductivity of the Sodium chloride solution (conductivity should be $16 \pm 0.2$ milli Siemens $(\mathrm{mS})$ at $\left.25^{\circ} \mathrm{C}\right)$

o White textile blotting paper or soft towels (Figure 1 \& 2).

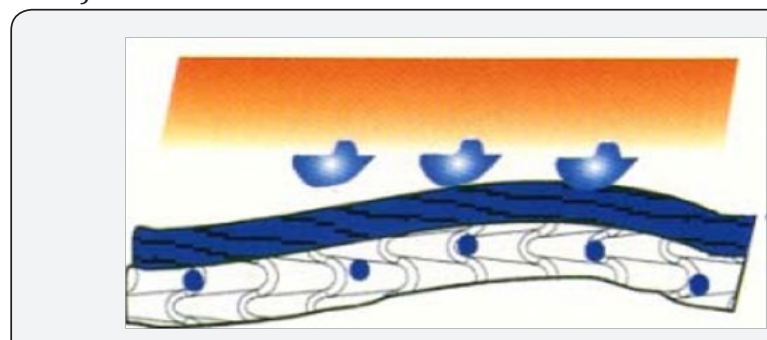

Figure 2: Moisture Management in Textiles.

Wetting time-WTT (Top) and WTB (Bottom): WTT and WTB are the time period in which the top and bottom surfaces of the fabric just start to get wetted respectively after the test commences, which are defined as the time in second (s) when the slope of total water contents at the top and bottom surfaces $\left(\mathrm{U}_{\text {top }}\right.$ and $\left.\mathrm{U}_{\text {bottom }}\right)$ become greater than tan $\left(15^{\circ}\right)$ respectively. Wetting time can be compared with the absorbency drop test specified in AATCC 79.

Absorption rate: TAR (Top) and BAR (Bottom): TAR and BAR are the average moisture absorption ability of the fabric top and bottom surfaces in the pump time respectively.

Maximum wetted radius: MWR (Top) and MWR (Bottom): Maximum wetted radii $\left(\mathrm{MWR}_{\text {top }}\right.$ and $\left.\mathrm{MWR}_{\mathrm{bottom}}\right)$ are defined as maximum wetted ring radius at the top and bottom surfaces respectively, where the slopes of total water content $\left(\mathrm{U}_{\text {top }}\right.$ or $\left.\mathrm{U}_{\text {bottom }}\right)$ become greater than $\tan \left(15^{\circ}\right)$ for the top and bottom surfaces respectively.

Spreading speed: TSS (Top) and BSS (Bottom): Spreading Speed is defined as the accumulative spreading speed from the centre to the maximum wetted radius.

Overall moisture management capacity (OMMC): Overall Moisture Management Capacity (OMMC) is an index to indicate the overall capability of the fabric to manage the transport of liquid moisture. Table 2 \& Figure 3 illustrates the grading of liquid moisture management properties of the tested sample.

Table 2: Grading of MMT Indices.

\begin{tabular}{|c|c|c|c|c|c|c|}
\hline \multirow{2}{*}{\multicolumn{2}{|c|}{ Index }} & \multicolumn{5}{|c|}{ Grade } \\
\hline & & 1 & 2 & 3 & 4 & 5 \\
\hline \multirow{4}{*}{ Wetting Time } & \multirow{2}{*}{ Top } & $\geq 120$ & $20-119$ & $5-19$ & $3-5$ & $<3$ \\
\hline & & No wetting & Slow & Medium & Fast & Very fast \\
\hline & \multirow{2}{*}{ Bottom } & $\geq 120$ & $20-119$ & $5-19$ & $3-5$ & $<3$ \\
\hline & & No wetting & Slow & Medium & Fast & Very fast \\
\hline \multirow{4}{*}{ Absorption rate } & \multirow{2}{*}{ Top } & $0-10$ & $10-30$ & $30-50$ & $50-100$ & $>100$ \\
\hline & & Very Slow & Slow & Medium & Fast & Very Fast \\
\hline & \multirow{2}{*}{ Bottom } & $0-10$ & $10-30$ & $30-50$ & $50-100$ & $>100$ \\
\hline & & Very Slow & Slow & Medium & Fast & Very Fast \\
\hline
\end{tabular}




\section{Current Trends in Fashion Technology \& Textile Engineering}

\begin{tabular}{|c|c|c|c|c|c|c|}
\hline \multirow{4}{*}{ Max wetted radius } & \multirow{2}{*}{ Top } & $0-7$ & $7-12$ & $12-17$ & $17-22$ & $>22$ \\
\hline & & No wetting & Small & Medium & Large & Very Large \\
\hline & \multirow{2}{*}{ Bottom } & $0-7$ & $7-12$ & $12-17$ & $17-22$ & $>22$ \\
\hline & & No wetting & Small & Medium & Large & Very Large \\
\hline \multirow{4}{*}{ Spreading Speed } & \multirow{2}{*}{ Top } & $0-1$ & $1-2$ & $2-3$ & $3-4$ & $>4$ \\
\hline & & Very Slow & Slow & Medium & Fast & Very Fast \\
\hline & \multirow{2}{*}{ Bottom } & $0-1$ & $1-2$ & $2-3$ & $3-4$ & $>4$ \\
\hline & & Very Slow & Slow & Medium & Fast & Very Fast \\
\hline \multirow{2}{*}{\multicolumn{2}{|c|}{ OWTC }} & $<-50$ & $-50-100$ & $100-200$ & $200-400$ & $>400$ \\
\hline & & Poor & Fair & Good & Very Good & Excellent \\
\hline \multirow{2}{*}{\multicolumn{2}{|c|}{ OMMC }} & $0-0.2$ & $0.2-0.4$ & $0.4-0.6$ & $0.6-0.8$ & $>0.8$ \\
\hline & & Poor & Fair & Good & Very Good & Excellent \\
\hline
\end{tabular}
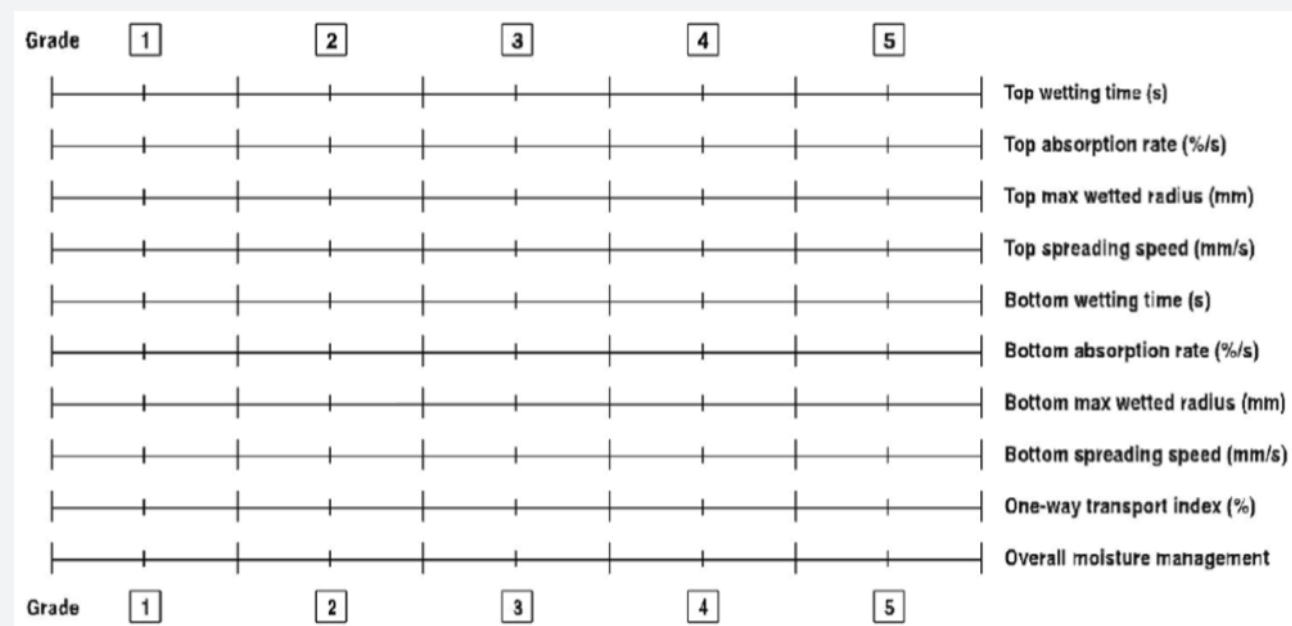

Figure 3: Grading of MMT Indices.

\section{Application of moisture management technique}

o Inner wears

o Athletic wear (active sportswear)

o Performance wear (climbing, walking, skiing)

o $\quad$ Comfort wear (nightwear)

o Military (multi-climate clothing)

o Health (hospital bed linens, wound dressings)

o Agricultural technology (Geo-textiles, greenhouse screening panels, soil moisture control)

o Technical solutions (Formula 1 protective clothing, fire fighting, industrial clothing)

o Industrial (filter \& valve technology, building, packaging)

o Upholstery (transport).

\section{Conclusion}

For comfort properties of textiles with varying end use applications, in the normal textile sector, technical textiles and other fields, moisture management plays a key role. Based on moisture management, textiles can be made tailor made for its specific end use. Apparel manufacturers are shifting their attention to the high-performance end use of the moisture management fabrics. As manufacturers of sports and active outdoor wear strive to improve the functionality of their collections, the future will see further developments in the field of moisture management fabrics.

\section{References}

1. Horrocks AR, Anand SC (2000) Handbook of Technical Textiles. Woodhead Publishing Ltd., UK Chapter 1, p. 1-23.

2. (2013) Book of Papers, National Seminar on "SPORTECH-Needs, Challenges \& Prospects" organized by the Wool Research Association, Thane held at the Pragati Maidan, New Delhi, India.

3. Petry GmbH Textilchemie.

4. MMT Testing instrument SDL Atlas, Machine manual, available at Wool Research Association (WRA), Thane.

5. Das A, Manshahia M (2014) High active sportswear - A critical review. Indian Journal of Fiber \& Textile Research 39(4): 441-449.

6. Uttam D (2013) Active Sportswear Fabrics. International Journal of IT, Engineering and Applied Sciences Research 2(1): 34-40. 


\section{Current Trends in Fashion Technology \& Textile Engineering}

7. Chinta SK, Gujar PD (2013) Significance of Moisture Management for High Performance Textile Fabrics. International Journal of Innovative Research in Science, Engineering and Technology 2(3): 814 -819.

8. Agrawal Y, Jaiswal H, Kapoor R Moisture Management.

9. Wallace M (2002) 100\% Moisture Management. Journal of Textile and Apparel Technology and Management 2(3)

10. Bagherzadeh R, Gorji M, Latifi M, Payvandy P, Kong LX (2012) Evolution of moisture management behavior of high-wicking 3D warp knitted spacer fabrics. Fibers and Polymers 13(4): 529-534.
11. Wang S, Li Y, Tokura H, Hu J, Kwok Y, et al. (2008) Effect of Phase Change Materials on Temperature and Moisture Distributions in Clothing during Exercise in Cold Environment. Journal of Fiber Bioengineering and Informatics 1(1): $29-40$

12. http://www.innovationintextiles.com/pine-cone-effect-to-be-usedin-moisture-management-fabrics/.

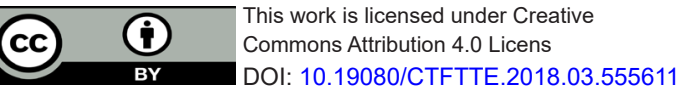

Your next submission with Juniper Publishers will reach you the below assets

- Quality Editorial service

- Swift Peer Review

- Reprints availability

- E-prints Service

- Manuscript Podcast for convenient understanding

- Global attainment for your research

- Manuscript accessibility in different formats

( Pdf, E-pub, Full Text, Audio)

- Unceasing customer service

Track the below URL for one-step submission https://juniperpublishers.com/online-submission.php 